\title{
Early Intervention as an Agent for Recovery of Function After Spinal Cord Injury- A Single Case Study
}

\author{
Priti Agni, Ankita Bhanushali \\ Department of Neurophysiotherapy, K. J. Somaiya College of Physiotherapy, Mumbai, India \\ Email address: \\ pritikulkarni2002@yahoo.co.uk (P.Agni),bhanushali.ankita95@gmail.com (A. Bhanushali)
}

To cite this article:

Priti Agni, Ankita Bhanushali. Early Intervention as an Agent for Recovery of Function After Spinal Cord Injury- A Single Case Study. International Journal of Neurologic Physical Therapy. Vol. 4, No. 2, 2018, pp. 34-39. doi: 10.11648/j.ijnpt.20180402.12

Received: April 22, 2018; Accepted: May 21, 2018; Published: July 3, 2018

\begin{abstract}
This case study describes a task-specific training program for working and functional recovery in a young man with incomplete cervical spinal cord injury. The subject was 20 year old male with traumatic spinal cord injury after a fall from height. Prior to intervention, he was quadriplegic with severe motor and sensory impairment. Our goal was to recover the function. The subject underwent disectomy for anterolisthesis of C6 over C7. Acute spinal cord management was started followed by locomotor training, electrical stimulation, orthoses, specialised assistive devices. Outcome measures used were ASIA scale, walking index for SCI, SCIM, Fatigue severity scale, Beck depression scale, SCI-FAI. It was found that specific interventions were intentionally selected in the development of treatment program which helped in the functional recovery of the patient. After 3 and half months treatment, the subject was able to walk with the assistance of AFOs and walker, and was able to perform ADLs independently. The magnitude of his functional improvements could be attributed to early intervention and neuroplasticity.
\end{abstract}

Keywords: Rehabilitation, Spinal Cord Injury, Quadriplegia, Task Specific Intervention

\section{Introduction}

The spinal cord extends from medulla oblongata just above the foramen magnum to the level of the L1 or L2 vertebra. Its tapered caudal end is called the conus medullaris [1].

The spinal cord contains long axons of upper motor neurons descending from brain, lower motor neurons (somatic motor neurons) with axons that travel to the periphery to innervate skeletal muscles, sympathetic and parasympathetic visceral motor neurons, long axons of sensory neurons ascending to the brain, and interneurons (segmental and intersegmental) that interconnect neurons within the cord itself. Normal voluntary and reflexive motor function, sensory function, and automatic control involve complex interactions between supraspinal systems, lower motor neurons, visceral motor neurons, peripheral afferents, and networks of interneurons. The spinal cord also contains glial cells, nonneuronal cells that are essential to the neurons' functioning and survival [1].

Spinal cord injury is a relatively low incidence high-cost injury that results in tremendous change in individual's life. Paralysis of the muscles below the level of injury can lead to limited and altered mobility, self care and ability to participate in valued social activities. In addition to the musculoskeletal system, many other body systems are impaired after SCI, including the cardiopulmonary, integumentary, genitourinary and sensory systems [2].

A literature search was performed in July 2012, according to that the incidence of SCI in developing countries is $25.5 / \mathrm{million} /$ year and ranges from 2.1 to $130.7 / \mathrm{million} /$ year. Males comprised $82.8 \%$ of all SCIs with a mean age of 32.4 years. The two leading causes of SCI we're found to be motor vehicle and falls [3].

A fall from height is the commonest mode of sustaining a spinal cord injury in developing countries. Spinal injuries are classified on the basis of mechanism of injury into the following type: flexion injury, flexion-rotation injury, vertical compression injury, extension injury, flexion- distraction injury, direct injury, indirect injury due to violent muscle contraction [4].

Life expectancy has increased over the years but is still less than that for individuals without a SCI. Factors that influence life expectancy are age at onset and level and extent of neurological injury. Individual with an incomplete 
neurological SCI have a longer life expectancy than those with complete injury, and individuals with more caudal injuries also have greater life expectancy. The financial impact of SCI is extremely high. Spinal cord injury is characterised by lengthy hospitalization, medical complications, extensive follow up care, and recurrent hospitalizations. SCI is a relatively low-incidence disability affecting predominantly younger males, although more older adults are experiencing SCI likely due to falls, and SCI is associated with lengthy and costly care [2].

Rehabilitation is an important element toward achieving a fulfilling and active life after SCI. Physical therapists play a key role in the rehabilitation process. The overarching goal of physical rehabilitation is for the patient to become as independent as possible and to achieve the functional mobility necessary for everyday living, works, and recreation. Activity dependent plasticity might help in early recovery in SCI patients [2]. Studies have shown rehabilitation interventions cause activation of spinal pathways above and below the level of injury. This increase in neural activity is thought to be beneficial towards the restoration of non functioning neural pathways and promotion of new connections. [5]

\section{Case Description}

A 20 year old boy was brought to the hospital after a fall from a height of 15-20 ft while playing traditional Indian festival (dahi handi). Patient complained of pain in posterior aspect of neck region with upper limb and lower limb weakness along with sensory loss below the level of umbilicus. Patient was operated for anterolisthesis of C6 over C7 via anterior cervical disectomy with plate fixation on $17 / 08 / 2017$. MRI findings showed post operative changes in the cervical spine at C6, C7 and D1 level in the form of internal fixation with metallic implants, causing susceptibility artifacts with mild anterior subluxation of the left $\mathrm{C} 6-\mathrm{C} 7$ facetal joint. A focal T2 hypertensive, T1 hypotensive non enhancing lesion in the cord at C6-C7 level, predominantly involving the dorsal cord- possibly post traumatic early myelomalacic change. He was started on physiotherapy thereafter and there was significant improvement in his outcome measures.

At the time of initial evaluation post surgery patient's sensory level was T4, motor level was $\mathrm{C} 4$ and neurological level was $\mathrm{C} 4$, with severe postural hypotension and no control of bowel and bladder. Patient was completely dependent with respect to transitions and ADLs. He received an extensive physical therapy consisting of acute spinal cord management, positioning, electrical stimulation, chest care, progressing to tilt table standing followed by active rehabilitation involving functional training, transitional training, locomotor training.

\section{Examination}

Assessments were performed by a physical therapist. Examination consisted of assessment of vitals, sensations, reflexes, motor examination including skin condition, muscle girth, range of motion, tightness, tone, involuntary movements, strategy assessment (once patient started sitting), gait assessment (once he started walking), outcome measures (ASIA scale [6], walking index for SCI [7], SCIM [8], Fatigue severity scale [9], Beck depression scale [10], SCIFAI [11]).

Patient presented with hypotension- BP 110/70 mm Hg, impairment of sensation such pain and touch which were intact at C2-C6 level, impaired at T4-T6 level and from T7 onwards it was absent, deep tendon reflexes were diminished, upper limb muscles were hypotonic while lower limb muscles had some tone in hip extensors, knee flexors and ankle clonus was present bilaterally, bowel bladder control was not there, patient was completely bed bound.

At the end of 3 and half month of treatment re-evaluation was performed which showed improvement such that BP came to normal $(120 / 80 \mathrm{~mm} \mathrm{Hg})$, sensations were intact everywhere, deep tendon reflexes become normal in upper limb and brisk in lower limb, tone of upper limb came to normal and in lower limb reduction of tone from grade 2 to grade 1 (Modified Ashworth Scale [12]) in most of the muscles, full control of bowel bladder achieved, strategy assessment and gait assessment was done as patient started sitting and walking.

Table 1. Outcome measures.

\begin{tabular}{|c|c|c|c|c|}
\hline Outcome measures & & Post surgery & After $1 \frac{1}{2}$ month of treatment & At the end of treatment \\
\hline \multirow{6}{*}{ ASIA scale } & 1. Motor & 1. & 1. & 1. \\
\hline & Upper limb & $11 / 50$ & $29 / 50$ & $38 / 50$ \\
\hline & Lower limb & $0 / 50$ & $14 / 50$ & $27 / 50$ \\
\hline & 2. Sensory & 2. & 2. & 2. \\
\hline & Light touch & $26 / 56$ & $37 / 56$ & $55 / 56$ \\
\hline & Pin prick & $26 / 56$ & $37 / 56$ & $55 / 56$ \\
\hline SCIM & & $8 / 100$ & $18 / 100$ & $67 / 100$ \\
\hline Beck depression scale & & $38 / 40$ & $26 / 40$ & $13 / 40$ \\
\hline \multirow{3}{*}{ SCI-FAI } & 1. Parameter & \multirow{3}{*}{$\begin{array}{l}\text { No } \\
\text { ambulation }\end{array}$} & $1.11 / 20$ & $1.18 / 20$ \\
\hline & 2. Assistive devices & & 2. $2 / 14$ & $2.4 / 14$ \\
\hline & 3. Temporal/ distance measures & & $3.1 / 5$ & $3.4 / 5$ \\
\hline Walking index for SCI & & Level 0 & Level 9 & Level 9 \\
\hline
\end{tabular}




\section{Intervention}

For first 2 weeks after surgery the treatment consisted of the following:

Positioning of patient to prevent contracture and to maintain skin integrity

Passive range of motion exercises of both upper limb and lower limb to maintain joint integrity

Chest percussion, vibrations and suctioning to prevent cardiorespiratory complications

Intermittent Galvanic Stimulation of lower limb muscles to improve muscle tone. Electrical stimulation of lower limb has been reported to increase muscle hypertrophy, prevent disuse atrophy and increase vascularisation. [14, 15]

From the third week onwards treatment was aimed at the following:

To promote early weight bearing and prevent postural hypotension tilt table standing was started with AFO's, upto $40^{\circ}-60^{\circ}$ inclination. Crepe bandage was applied to prevent peripheral pooling. Weight bearing activities have shown to slow the loss of bone during acute stage, decrease extensor spasm and improve overall health. [16]

To initiate bed mobility exercises Therapist assisted bridging and rolling were started.

To improve upper limb strength, upper limb active exercises were given.

From four to six weeks treatment consisted of:

To improve upper limb strength multiple angle isometrics were done and to initiate lower limb mobility active assisted range of motion exercises were given. Patient was switched from Intermittent galvanic stimulation to strong surge faradic stimulation for quadriceps, dorsiflexors, lumbricals and interossei of hand. Gripping exercises with soft ball to improve grip was done.

Bed mobility exercises consisted of independent rolling and side lying to sit.

Tilt table standing progressed upto 90 degrees.

To train sitting and standing balance, sitting maintenance exercises such as reach outs, weight shift, push ups in sitting. Standing maintenance followed by standing balance exercises was done. Sit to stand with KAFOs and walker in front was done.

From seven to nine weeks treatment consisted of:

To make patient ambulatory, with assistance pre gait training including swing to, swing through gait, jack knifing gait training was done. Gait training in parallel bars with KAFOs was carried out. As patient ambulated, the therapist facilitated the movement of each leg while stabilising the trunk. Each therapist's hand placements facilitated muscle activation and completion of desired activity. Gait training is thought to activate the CPG. $[2,13,18]$

To achieve the functional level of Independence, independent bed mobility manoeuvres such as rolling, prone on elbows, supine to sit was carried out. Transfer training included wheelchair transfer from bed and wheelchair training.

From nine to twelve weeks treatment consisted of:
To allow the individual to return to his normal roles and fully participate in society, activity dependent upper limb training, independent transfer - from chair to chair, chair to vehicle, community walking - walking with walker and AFOs and Stair climbing was carried out.
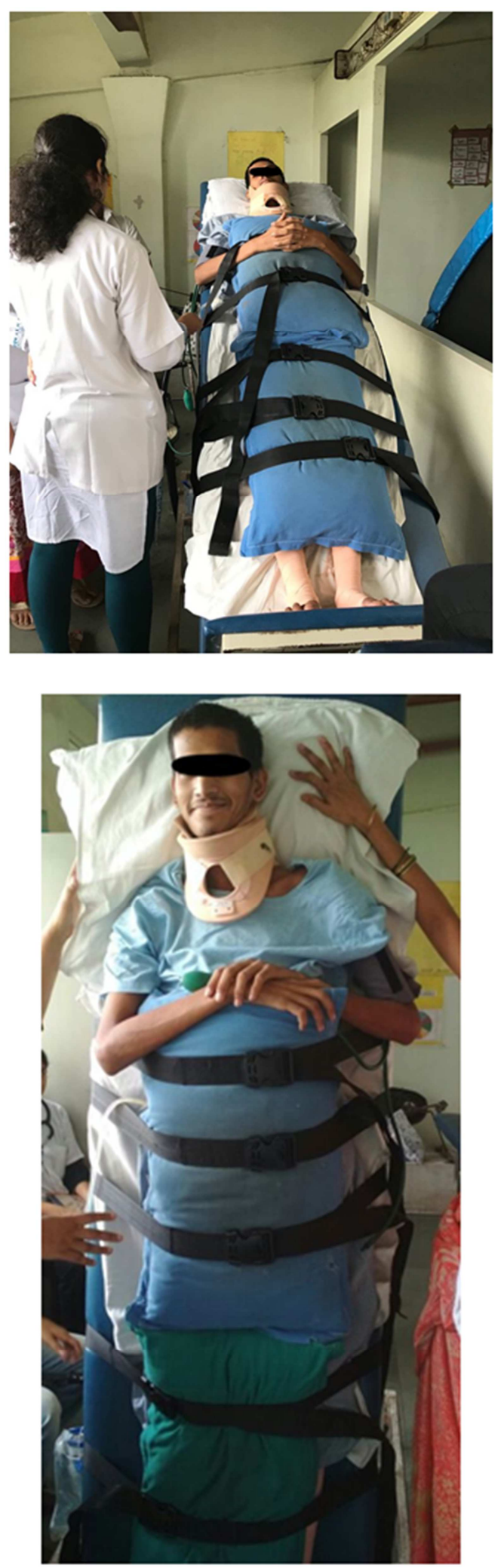

Figure 1. Tilt table standing. 

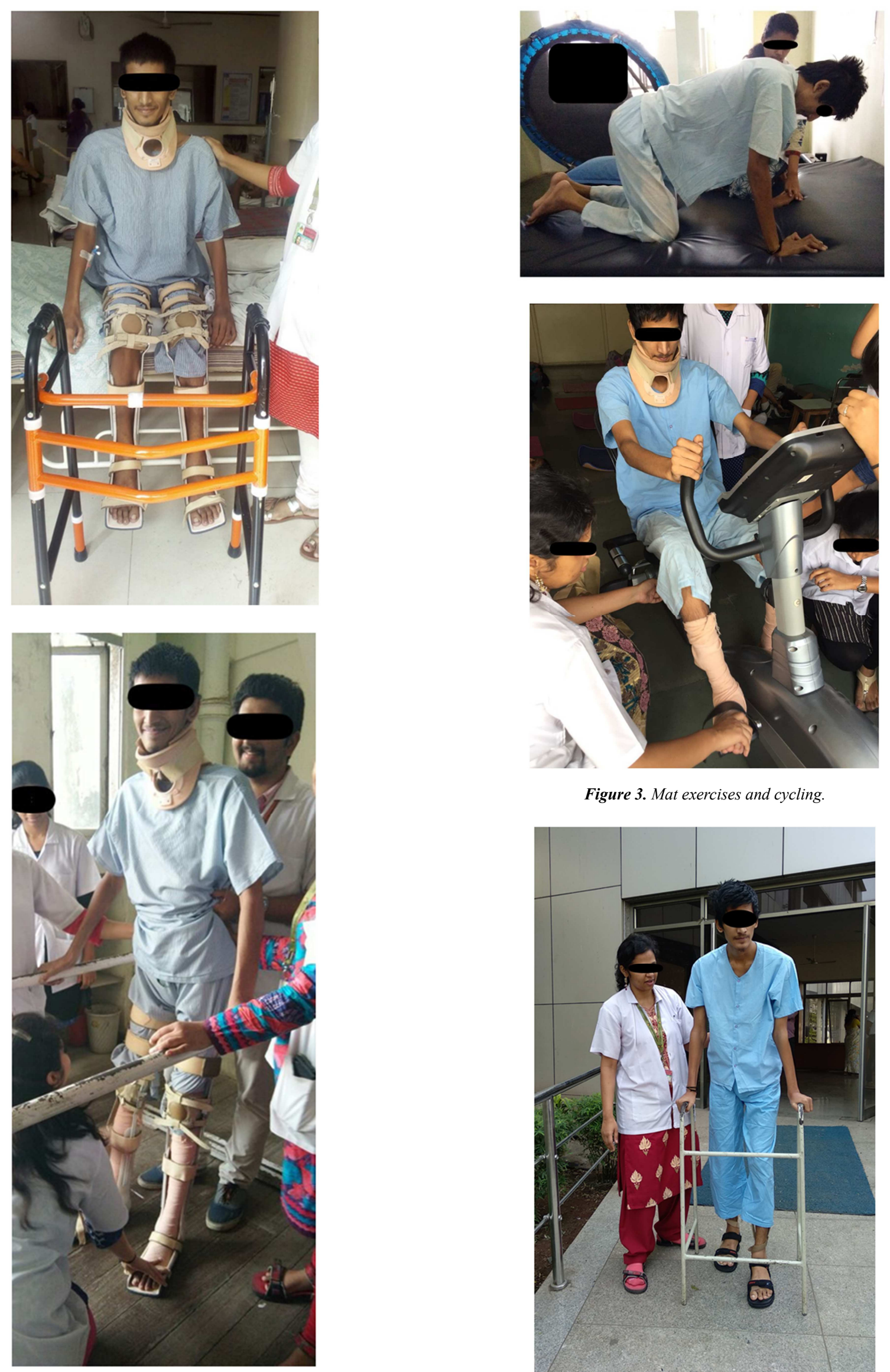

Figure 3. Mat exercises and cycling.

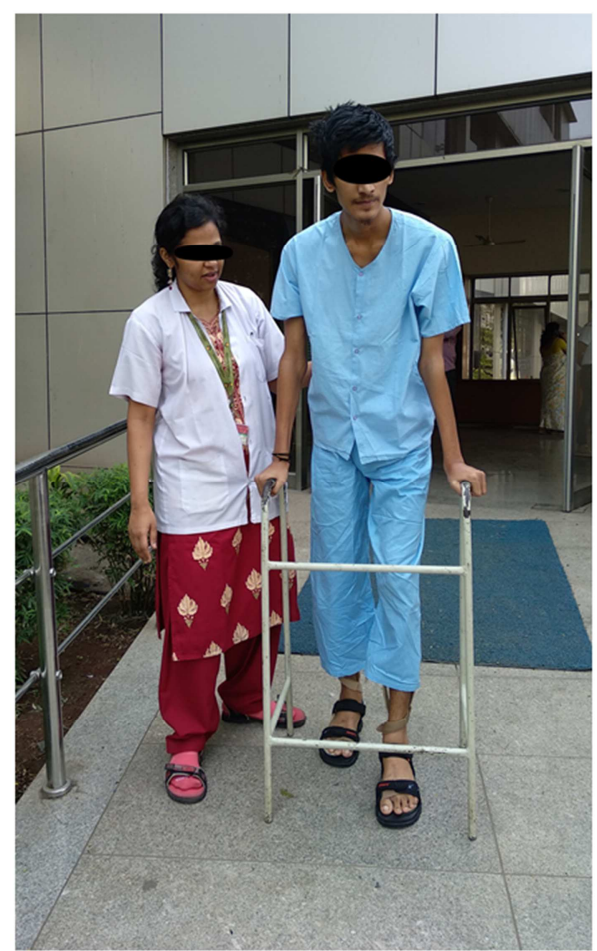

Figure 2. Sitting and standing balance. 


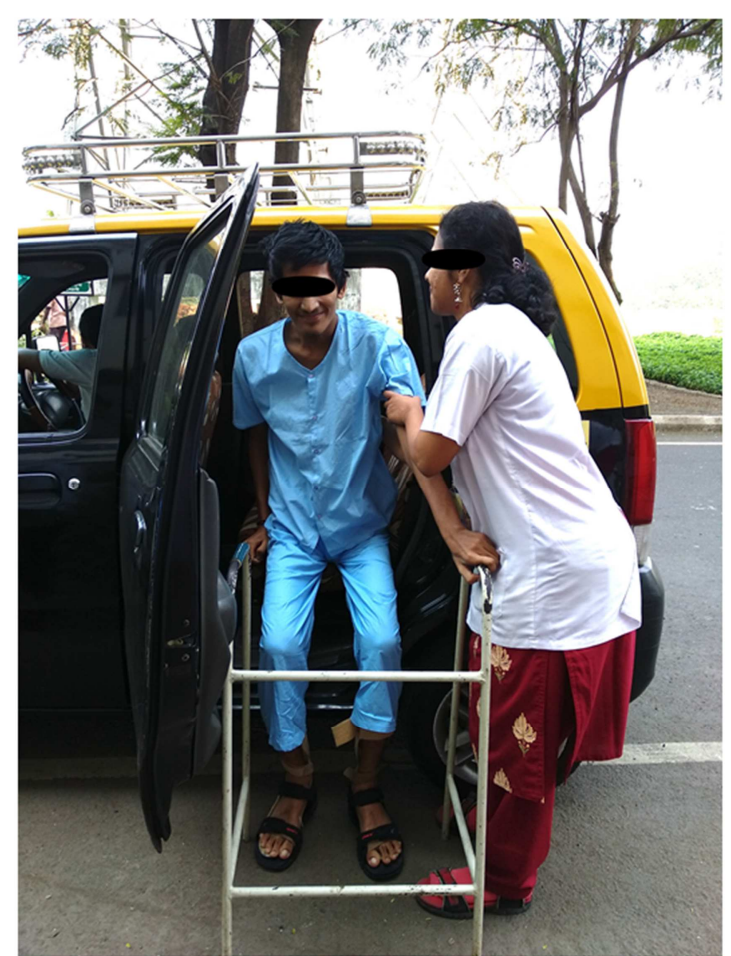

Figure 4. Community ambulation and vehicle transfer.

\section{Discussion}

This case study describes a holistic treatment plan designed to promote functional recovery in a young man with a spinal cord injury. This case Chronicles the recovery of an individual with severe impairments that might not have been expected given the chronicity of his injury. There have been very few published reports of individuals with such a high spinal cord injury (C6 level) who are able to walk. Expected recovery in C6 level injury is wheelchair ambulation but our patient made significant function gains over the course of therapy as he was subjected to early intervention. [2, 13]

Patient received early rigorous acute spinal cord management including positioning, bed mobility, electrical stimulation, chest care, transfer training, early weight bearing activities, tilt table standing and progressing to exercises to improve lower limb voluntary control, over ground ambulation training, cycling. The ASIA scale score was improved from motor-11/100, sensory- 52/112 ( post surgery) to motor-65/100, sensory-110/112 (after 3 and half month of treatment), improvement was also seen in functional Independence as SCIM score was 8/100 post surgery and later it became $67 / 100$ after treatment. This motor and functional recovery is due to early interventions, activity based restorative therapies which led to spontaneous plasticity. [1, 5] Spontaneous plasticity after spinal cord injury includes structural changes such as axonal sprouting in both damaged and undamaged neurons projecting to and from the brain, axonal sprouting in spared intersegmental interneurons, creation of new synapses, and synaptic remodeling [1]. Task specific practice is thought to be imperative to the development of skilled movements. The key to learning any skilled movements begins with repetition of the same movement over and over to develop motor pattern.[5] A study done by Tse CM concluded that task specific training interventions revealed positive effects on sitting and standing balance.[17] Task specific training was given to patient such as activity dependent upper limb and lower limb training, gait training using appropriate orthoses and functional stimulation showed changes in score of Walking Index Scale from level 0 to level 9 and in SCI-FAI from no ambulation to $26 / 49$, so this task specific training may have facilitated better walking practice activity. Locomotion represents the interaction between the innate pattern and an appropriate modulation of leg muscles activation which has to continuously adapt to the present requirements. Feedback from variety of sources eg. visual, vestibular and proprioceptive is interpreted and then integrated into activity of CPG. The sensory feedback and context specific requirements of motor tasks determine the mode of organisation of muscle synergies. [18] Activitydependent plasticity occurs in response to afferent input. Sensory input from the periphery, typically arising from repetitive limb activity, causes adaptive neuronal changes. As activity dependent plasticity is task specific, the performance of that activity was enhanced and will not generalize to others [1]. Hence patient was trained for UL strengthening, mat exercises, exercises to improve bowel and bladder control. Repetition and intensity are both critical for driving plasticity and learning.[19] This also helped in improving endurance and reducing fatigue caused improvement of score of Fatigue Severity Scale from 55/63 to 35/63. Massed practice was vital component of program as the number of repetitions and the extended amount of activity time is thought to be crucial in initiating positive results by probably leading to remyelination or developing new neuronal connections. [5] Extensive physiotherapy treatment was given to patient for around 4 hours a day by therapist. He used to perform exercises 2 hours a day with the help of his caregiver. This type of structured practice, repetition and intensity is essential to optimise outcomes.

Because of the devastating nature of spinal cord injury primary focus was to remain positive and enthusiastic towards the task at hand and promoting achievement of rehabilitation goal. The radiation of this attitude and demeanour towards the patient encouraged high morale and determination. As the patient was motivated compliance improved. Simulation of task such as eating, bathing was taught which helped in improving functional capabilities. Combination of neuroprotective, neuroregenerative and neurorehab strategies are most likely to be effective moving forward given the multifaceted nature of injury. [20]

\section{Conclusion}

The landscape of spinal cord injury management is quickly changing as the heterogeneity of patients and long term importance of key early interventions are increasingly being recognised. Designing effective neuro rehabilitation after 
spinal cord injury depends on having knowledge about neuronal mechanism, interaction between central programs, afferent feedback and neural control of locomotion. Specific multi-pronged neuro rehabilitation approaches will pave the way for more effective strategies to improve motor function and functional performance in patients with spinal cord injury. Hence it was concluded that specific early interventions which were intentionally selected helped in functional recovery of the patient. The transition of patient from being functionally dependent to being able to walk with assistance of AFOs and walker and becoming functionally independent was attributed to task specific training, early intervention and neuroplasticity.

\section{References}

[1] Somers MF: Spinal Cord Injury Functional Rehabilitation, 3rd Ed. Prentice Hall; 2001

[2] O'Sullivan SB, Schmitz TJ. Traumatic spinal cord injury in Physical Rehabilitation, F. A. Davis, Philadelphia. 6th ed, 2014:889-963.

[3] Rahimi-Movaghar V et al. Epidemiology of traumatic SCI in developing countries: A systemic review, Neuroepidemiology 2013 Jun; 41(2): 65-85.

[4] Maheshwari and Mhaskar: Essential Orthopaedics (including clinical methods), Jaypee Publications. 5th ed, 2015:267.

[5] Dolbow DR, Gorgey AS, McDonald JW: Activity based restorative therapies after spinal cord injuries: Interinstitutional conceptions and perceptions, Ageing Dis. 2015 Aug; 6(4): 254-261.

[6] Steven C. Kirshblum, Stephen P. Burns, Fin Biering-Sorensen, William Donovan, Daniel D. Graves, Amitabh Jha, et al: International standards for neurological classification of spinal cord injury (revised 2011). J Spinal Cord Med. 2011 Nov; 34(6): 535-546.

[7] Ditunno JF Jr, Ditunno PL, Graziani V, Scivoletto G, Bernardi $\mathrm{M}$, Castellano V, et al: Walking Index for spinal cord injury (WISCI): an international multicenter validity and reliability study, Spinal Cord. 2000 Apr;38(4):234-43.

[8] Itzkovich M., Gelernter I., Viering-Sorensen F., Weeks C., Laramee MT., Carven BC, et al: The Spinal Cord Independence Measure (SCIM) version III: Reliability and validity in a multi-center international study, Disabil Rehabil. 2007 Dec 30; 29 (24):1926-33. Epub 2007 Mar 5.
[9] J. Scott, K. Rosa, M. Fu, K. Cerri, M. Peeters, M. DeumoneMauviel, et al: Fatigue Severity Scale: Reliability, Validity and Interpretation Of Change- Evidence From Two Clinical Trials In Patients with Chronic Hcv Infection, 2013.

[10] Kuhner C, Burger C, Keller F, Hautzinger M: Reliability and validity of the Revised Beck Depression Inventory (BDI- 11). Results from German samples. Nervenarzt. 2007 Jun; 78(6):651-6.

[11] Edelle C. Field-Fote, Gerard G. Fluet, Scott D. Schafer, Eric M. Schneider, Robin Smith, Pamela A. Downey, et al: The Spinal Cord Injury Functional Ambulation Inventory (SCIFAI). J Rehabilitation Med. 2001 Jul; 33 (4):177-81.

[12] Craven BC, Morris AR: Modified Ashworth Scale Reliability for measurement of lower extremity spasticity among patients with SCI. Spinal Cord. 2010 Mar; 48 (3):207-13.

[13] Ida Bromley. Tetraplegia and paraplegia -A guide for physiotherapist. Churchill Livingstone. 6th Ed, July 2006.

[14] Ho CH, Triolo RJ, Elias AL, Kilgore KL, DiMarco AF, Bogie $\mathrm{K}$ et al: Functional electrical stimulation and spinal cord injury. Phys Med RehabilnClin N Am. 2014 Aug; 25 (3).

[15] Martin R, Sadowsky C, Obst K, Brooke M, McDonald J. Functional Electrical Stimulation in SCI - From theory to practice. Top Spinal Cord Inj Rehabil. 2012; 18(1): 28-33.

[16] Adams MM, Hicks Al: Comparison of effects of body weight supported treadmill training and tilt table standing spasticity in Individuals with chronic spinal cord injury. J Spinal Cord Med 2011; 34(5): 488-494.

[17] Tse CM, Chisholm AE, Lam T, Eng JJ: A systematic review of the effectiveness of task specific rehabilitation interventions for improving independent sitting and standing function in spinal cord injury. J Spinal Cord Med 2018; 41(3): 254-266.

[18] Hubli M, Dietz V. The physiological basis of neurorehabilitation locomotor training after spinal cord injury. Journal of Neuroengineering and Rehabilitation. 2013; 10:5.

[19] Cook AS, Woollacott MH. Motor learning and recovery of function in Motor control theory and practical applicationtions. Lippincott Williams \& Wilkins. 5th Ed; 2016.

[20] Ahuja CS, Martin AR, Fehlings M. Recent advances in managing Spinal cord injury secondary to trauma. F1000 Research 2016; 5 (F1000 Faculty Rev): 1017. 